\title{
Well-posed generalized vector equilibrium problems
}

\author{
Xicai Deng ${ }^{1,2^{*}}$ and Shuwen Xiang ${ }^{2}$
}

${ }^{*}$ Correspondence:

iamdengxicai@163.com

'Department of Mathematics and Computer, Guizhou Normal College,

Guiyang, 550018, China

${ }^{2}$ Department of Mathematics,

Guizhou University, Guiyang,

550025, China

\begin{abstract}
In this paper, we establish the bounded rationality model $M$ for generalized vector equilibrium problems by using a nonlinear scalarization technique. By using the model $M$, we introduce a new well-posedness concept for generalized vector equilibrium problems, which unifies its Hadamard and Levitin-Polyak well-posedness. Furthermore, sufficient conditions for the well-posedness for generalized vector equilibrium problems are given. As an application, sufficient conditions on the well-posedness for generalized equilibrium problems are obtained.
\end{abstract}

MSC: 49K40; 90C31

Keywords: well-posedness; generalized vector equilibrium problems; bounded rationality model; nonlinear scalarization function

\section{Introduction}

As is well known, the notion of well-posedness can be divided into two different groups: Hadamard type and Tykhonov type [1]. Roughly speaking, Hadamard types of wellposedness for a problem means the continuous dependence of the optimal solution from the data of the problem. Tykhonov types of well-posedness for a problem such as Tykhonov and Levitin-Polyak well-posedness are based on the convergence of approximating solution sequences of the problem. Some researchers have investigated the relations between them for different problems (see [1-4]). The notion of extended wellposedness has been proposed by Zolezzi [5] in the context of scalar optimization. In some sense this notion unifies the ideas of Tykhonov and Hadamard well-posedness. Moreover, the notion of extended well-posedness has been generalized to vector optimization problems by Huang [6-8].

On the other hand, the vector equilibrium problem provides a very general model for a wide range of problems, for example, the vector optimization problem, the vector variational inequality problem, the vector complementarity problem and the vector saddle point problem. In the literature, existence results for various types of vector equilibrium problems have been investigated intensively; see, e.g., $[9,10]$ and the references therein. The study of well-posedness for vector equilibrium problems is another important topic in vector optimization theory. Recently, Tykhonov types well-posedness for vector optimization problems, vector variational inequality problems and vector equilibrium problems have been intensively studied in the literature, such as [11-17]. Among those papers, we observe that the scalarization technique is an efficient approach to deal with Tykhonov types well-posedness for vector optimization problems. As noted in $[12,16]$, the notions of 
well-posedness in the scalar case can be extended to the vector case and, for this end, one needs an appropriate scalarizarion technique. Such a technique is supposed to preserve some well-posedness properties when one passes from the vectorial to the scalar case, and simple examples show that linear scalarization is not useful from this point of view even in the convex case. An effort in this direction was made in the papers (see $[11,12,16,18]$ ). Miglierina et al. [11] investigated several types of well-posedness concepts for vectorial optimization problems by using a nonlinear scalarization procedure. Some equivalences between well-posedness of vectorial optimization problems and well-posedness of corresponding scalar optimization problems are given. By virtue of a nonlinear scalarization function, Durea [12] proved the Tykhonov well-posedness of the scalar optimization problems are equivalent to the Tykhonov well-posedness of the original vectorial optimization problems. Very recently, Li and Xia [16] investigated Levitin-Polyak well-posedness for vectorial optimization problems by using a nonlinear scalarization function. They also showed the equivalence relations between the Levitin-Polyak well-posedness of scalar optimization problems and the vectorial optimization problems.

Motivated and inspired by the research work mentioned above, we introduce a new wellposedness concept for generalized vector equilibrium problems (in short (GVEP)), which unifies its Hadamard and Levitin-Polyak well-posedness. The concept of well-posedness for (GVEP) is investigated by using a new method which is different from the ones used in [5-8]. Our method is based on a nonlinear scalarization technique and the bounded rationality model $M$ (see [19-22]). Furthermore, we give some sufficient conditions on various types of well-posedness for (GVEP). Finally, we apply these results to generalized equilibrium problems (in short (GEP)).

\section{Preliminaries}

Let $X$ be a nonempty subset of the Hausdorff topological space $H$ and $Y$ be a Hausdorff topological vector space. Assume that $C$ denotes a nonempty, closed, convex, and pointed cone in $Y$ with apex at the origin and int $C \neq \emptyset$, where int $C$ denotes the topological interior of $C$.

Let $G: X \rightrightarrows X$ be a set-valued mapping and $\varphi: X \times X \rightarrow Y$ be a vector-valued mapping, the problem of interest, called generalized vector equilibrium problems (in short (GVEP)), which consist of finding an element $x \in X$ such that $x \in G(x)$ and

$$
\varphi(x, y) \notin-\operatorname{int} C, \quad \forall y \in G(x) .
$$

When $Y=\mathbb{R}$ and $C=[0,+\infty[$, the generalized vector equilibrium problem becomes the generalized equilibrium problem (in short (GEP)): finding an element $x \in X$ such that $x \in G(x)$ and

$$
\varphi(x, y) \geq 0, \quad \forall y \in G(x) .
$$

Now we introduce the notion of Levitin-Polyak approximating solution sequence for (GVEP).

Definition 2.1 A sequence $\left\{x_{n}\right\} \subset X$ is called a Levitin-Polyak approximating solution sequence (in short LP sequence) for (GVEP), if there exists $\left\{\epsilon_{n}\right\} \subset \mathbb{R}_{+}$with $\epsilon_{n} \rightarrow 0$ such 
that

$$
d\left(x_{n}, G\left(x_{n}\right)\right) \leq \epsilon_{n}
$$

and

$$
\varphi\left(x_{n}, y\right)+\epsilon_{n} e \notin-\operatorname{int} C, \quad \forall y \in G\left(x_{n}\right) .
$$

Next, we introduce a nonlinear scalarization function and their related properties.

Lemma $2.1([10,18,23])$ For fixed $e \in$ int $C$, the nonlinear scalarization function is defined by

$$
\xi_{e}(y)=\inf \{r \in \mathbb{R}: y \in r e-C\}, \quad \forall y \in Y .
$$

The nonlinear scalarization function $\xi_{e}$ has the following properties:

(i) $\xi_{e}(y) \geq r \Longleftrightarrow y \notin r e-$ int $C$;

(ii) $\xi_{e}(r e)=r$;

(iii) $\xi_{e}\left(y_{1}+y_{2}\right) \leq \xi_{e}\left(y_{1}\right)+\xi_{e}\left(y_{2}\right)$, for all $y_{1}, y_{2} \in Y$

Definition 2.2 Let $\varphi: X \rightarrow Y$ be a vector-valued mapping.

(i) $\varphi$ is said to be $C$-upper semicontinuous at $x$ if for any open neighborhood $V$ of zero element in $Y$, there is an open neighborhood $U$ at $x$ in $X$ such that for any $x^{\prime} \in U$, $\varphi\left(x^{\prime}\right) \in \varphi(x)+V-C ;$

(ii) $\varphi$ is said to be $C$-upper semicontinuous on $X$ if $\varphi$ is $C$-upper semicontinuous on each $x \in X$

(iii) $\varphi$ is said to be $C$-lower semicontinuous at $x$ if for any open neighborhood $V$ of zero element in $Y$, there is an open neighborhood $U$ at $x$ in $X$ such that for any $x^{\prime} \in U$, $\varphi\left(x^{\prime}\right) \in \varphi(x)+V+C ;$

(iv) $\varphi$ is said to be $C$-lower semicontinuous on $X$ if $\varphi$ is $C$-lower semicontinuous on each $x \in X$.

Remark 2.1 In Definition 2.2, when $Y=\mathbb{R}, C=[0,+\infty$ [, being $C$-upper semicontinuous reduces to being upper semicontinuous and being $C$-lower semicontinuous reduces to being lower semicontinuous.

Lemma 2.2 If $\varphi: X \times X \rightarrow Y$ is $C$-upper semicontinuous on $X \times X$, then $\xi_{e} \circ \varphi: X \times X \rightarrow \Re$ is upper semicontinuous on $X \times X$.

Proof In order to show that $\xi_{e} \circ \varphi: X \times X \rightarrow \Re$ is upper semicontinuous on $X \times X$, we must check, for any $r \in \Re$, the set $L=\left\{(x, y) \in X \times X: \xi_{e}(\varphi(x, y)) \geq r\right\}$ is closed.

Let $\left(x_{n}, y_{n}\right) \in L$ and $\left(x_{n}, y_{n}\right) \rightarrow\left(x_{0}, y_{0}\right)$, we have $\xi_{e}\left(\varphi\left(x_{n}, y_{n}\right)\right) \geq r$, that is to say, by Lemma 2.1(i), $\varphi\left(x_{n}, y_{n}\right) \notin r e-\operatorname{int} C$. Next, we only need to prove that $\xi_{e}\left(\varphi\left(x_{0}, y_{0}\right)\right) \geq r$, that is, $\varphi\left(x_{0}, y_{0}\right) \notin r e-\operatorname{int} C$. By way of contradiction, assume that $\varphi\left(x_{0}, y_{0}\right) \in r e-\operatorname{int} C$, then there exists an open neighborhood $V$ of zero element in $Y$ such that

$$
\varphi\left(x_{0}, y_{0}\right)+V \subset r e-\operatorname{int} C
$$


Since $\varphi$ is $C$-upper semicontinuous at $\left(x_{0}, y_{0}\right) \in X \times X$, we have

$$
\varphi\left(x_{n}, y_{n}\right) \in \varphi\left(x_{0}, y_{0}\right)+V-C \subset r e-\operatorname{int} C-C \subset r e-\operatorname{int} C .
$$

It contradicts $\varphi\left(x_{n}, y_{n}\right) \notin r e-\operatorname{int} C$. So $L$ is closed. It shows $\xi_{e} \circ \varphi: X \times X \rightarrow \Re$ is upper semicontinuous on $X \times X$.

Finally, we recall some useful definitions and lemmas.

Let $F: X \rightrightarrows Y$ be a set-valued mapping. $F$ is said to be upper semicontinuous at $x \in X$ if for any open set $U \supset F(x)$, there is an open neighborhood $O(x)$ of $x$ such that $U \supset F\left(x^{\prime}\right)$ for each $x^{\prime} \in O(x)$; $F$ is said to be lower semicontinuous at $x$ if for any open set $U \cap F(x) \neq \emptyset$, there is an open neighborhood $O(x)$ of $x$ such that $U \cap F\left(x^{\prime}\right) \neq \emptyset$, for each $x^{\prime} \in O(x)$; $F$ is said to be an usco mapping if $F$ is upper semicontinuous and $F(x)$ is nonempty compact for each $x \in X ; F$ is said to be closed if $\operatorname{Graph}(F)$ is closed, where $\operatorname{Graph}(F)=\{(x, y) \in X \times Y$ : $x \in X, y \in F(x)\}$ is the graph of $F$.

Lemma 2.3 [22] Let $X$ and $Y$ be two metric spaces. Suppose that $F: Y \rightrightarrows X$ is a usco mapping. Then for any $y_{n} \rightarrow y$ and any $x_{n} \in F\left(y_{n}\right)$, there is a subsequence $\left\{x_{n_{k}}\right\} \subset\left\{x_{n}\right\}$ such that $x_{n_{k}} \rightarrow x \in F(y)$.

Lemma 2.4 [24] If $F: Y \rightrightarrows X$ is closed and $X$ is compact, then $F$ is upper semicontinuous on $Y$.

Let $(X, d)$ be a metric space. Denote by $K(X)$ all nonempty compact subsets of $X$. For arbitrary $C_{1}, C_{2} \subset X$, define

$$
h\left(C_{1}, C_{2}\right)=\max \left\{h^{0}\left(C_{1}, C_{2}\right), h^{0}\left(C_{2}, C_{1}\right)\right\},
$$

where

$$
h^{0}\left(C_{1}, C_{2}\right)=\sup \left\{d\left(b, C_{2}\right): b \in C_{1}\right\}
$$

and

$$
d\left(b, C_{2}\right)=\inf \left\{d(b, c), c \in C_{2}\right\} .
$$

It is obvious that $h$ is a Hausdorff metric on $K(X)$.

Lemma 2.5 [25] Let $(X, d)$ be a metric space and $h$ be Hausdorff metric on $X$. Then $(K(X), h)$ is complete if and only if $(X, d)$ is complete.

\section{Bounded rationality model and definition of well-posedness for (GVEP)}

Let $(X, d)$ be a metric space. The problem space $\Lambda$ of (GVEP) is given by

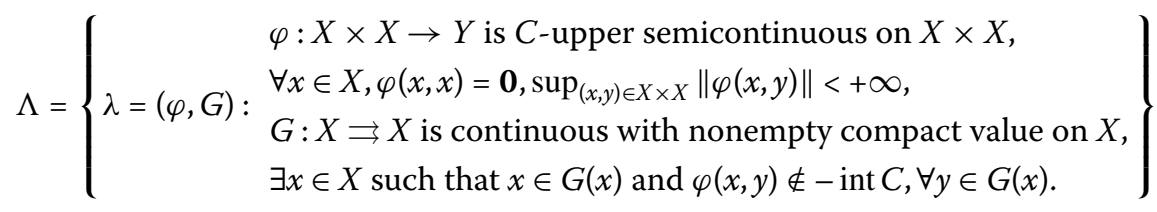


For any $\lambda_{1}=\left(\varphi_{1}, G_{1}\right), \lambda_{2}=\left(\varphi_{2}, G_{2}\right) \in \Lambda$, define

$$
\rho\left(\lambda_{1}, \lambda_{2}\right):=\sup _{(x, y) \in X \times X}\left\|\varphi_{1}(x, y)-\varphi_{2}(x, y)\right\|+\sup _{x \in X} h\left(G_{1}(x), G_{2}(x)\right),
$$

where $h$ denotes a Hausdorff distance on $X$. Clearly, $(\Lambda, \rho)$ is a metric space.

Next, the bounded rationality model $M=\{\Lambda, X, f, \Phi\}$ for (GVEP) is defined as follows:

(i) $\Lambda$ and $X$ are two metric spaces;

(ii) the feasible set of the problem $\lambda \in \Lambda$ is defined by

$$
f(\lambda):=\{x \in X: x \in G(x)\}
$$

(iii) the solution set of the problem $\lambda \in \Lambda$ is defined by

$$
E(\lambda):=\{x \in G(x): \varphi(x, y) \notin-\operatorname{int} C, \forall y \in G(x)\}
$$

(iv) the rationality function of the problem $\lambda \in \Lambda$ is defined by

$$
\Phi(\lambda, x):=\sup _{y \in G(x)}\left\{-\xi_{e}(\varphi(x, y))\right\}
$$

\section{Lemma 3.1}

(1) $x \in f(\lambda), \Phi(\lambda, x) \geq 0$.

(2) For all $\lambda \in \Lambda, E(\lambda) \neq \emptyset$.

(3) For all $\lambda \in \Lambda$ and $\epsilon \geq 0, \Phi(\lambda, x)=\sup _{y \in G(x)}\left\{-\xi_{e}(\varphi(x, y))\right\} \leq \epsilon$ if and only if $\varphi(x, y)+\epsilon e \notin-\operatorname{int} C, \forall y \in G(x)$. Particularly, $x \in E(\lambda)$ if and only if $\Phi(\lambda, x)=0$.

Proof (1) If $x \in f(\lambda)$, then $x \in G(x)$. By Lemma 2.1(i), we have

$$
\Phi(\lambda, x) \geq-\xi_{e}(\varphi(x, x))=0
$$

(2) Obvious

(3) If $\varphi(x, y)+\epsilon e \notin-\operatorname{int} C, \forall y \in G(x)$, by Lemma 2.1(i), $\xi_{e}(\varphi(x, y)) \geq-\epsilon, \forall y \in G(x)$. Thus, we have $\Phi(\lambda, x)=\sup _{y \in G(x)}\left\{-\xi_{e}(\varphi(x, y))\right\} \leq \epsilon$.

Conversely, if $\Phi(\lambda, x)=\sup _{y \in G(x)}\left\{-\xi_{e}(\varphi(x, y))\right\} \leq \epsilon$, then $\xi_{e}(\varphi(x, y)) \geq-\epsilon, \forall y \in G(x)$. By Lemma 2.1(i), we get $\varphi(x, y)+\epsilon e \notin-\operatorname{int} C, \forall y \in G(x)$.

Remark 3.1 By Definition 2.1 and Lemma 3.1, for all $\epsilon_{n}>0$ with $\epsilon_{n} \rightarrow 0$, the set of LP approximating solution for the problem $\lambda$ is defined as

$$
E\left(\lambda, \epsilon_{n}\right):=\left\{x \in X: d(x, f(\lambda)) \leq \epsilon_{n}, \Phi(\lambda, x) \leq \epsilon_{n}\right\}
$$

the set of solutions for the problem $\lambda$ is defined as

$$
E(\lambda)=E(\lambda, 0):=\{x \in X: x \in f(\lambda), \Phi(\lambda, x)=0\} .
$$

Hence, Levitin-Polyak well-posedness for (GVEP) is defined as follows. 


\section{Definition 3.1}

(i) If $\forall x_{n} \in E\left(\lambda, \epsilon_{n}\right), \epsilon_{n}>0$ with $\epsilon_{n} \rightarrow 0$, there must exist a subsequence $\left\{x_{n_{k}}\right\} \subset\left\{x_{n}\right\}$ such that $x_{n_{k}} \rightarrow x \in E(\lambda)$, then the problem $\lambda \in \Lambda$ is said to be generalized Levitin-Polyak well-posed (in short GLP-wp);

(ii) If $E(\lambda)=\{x\}$ (a singleton), $\forall x_{n} \in E\left(\lambda, \epsilon_{n}\right), \epsilon_{n}>0$ with $\epsilon_{n} \rightarrow 0$, there must have $x_{n} \rightarrow x$, then the problem $\lambda \in \Lambda$ is said to be Levitin-Polyak well-posed (in short LP-wp).

Referring to [3], Hadamard well-posedness for (GVEP) is defined as follows.

\section{Definition 3.2}

(i) If $\forall \lambda_{n} \in \Lambda, \lambda_{n} \rightarrow \lambda, \forall x_{n} \in E\left(\lambda_{n}\right)$, there must exist a subsequence $\left\{x_{n_{k}}\right\} \subset\left\{x_{n}\right\}$ such that $x_{n_{k}} \rightarrow x \in E(\lambda)$, then the problem $\lambda \in \Lambda$ is said to be generalized Hadamard well-posed (in short GH-wp);

(ii) If $E(\lambda)=\{x\}$ (a singleton), $\forall \lambda_{n} \in \Lambda, \lambda_{n} \rightarrow \lambda, \forall x_{n} \in E\left(\lambda_{n}\right)$, we must have $x_{n} \rightarrow x$, then the problem $\lambda \in \Lambda$ is said to be Hadamard well-posed (in short H-wp).

Next, we establish a new well-posedness concept for (GVEP), which unifies its Hadamard and Levitin-Polyak well-posedness.

\section{Definition 3.3}

(i) If $\forall \lambda_{n} \in \Lambda, \lambda_{n} \rightarrow \lambda, \forall x_{n} \in E\left(\lambda_{n}, \epsilon_{n}\right), \epsilon_{n}>0$ with $\epsilon_{n} \rightarrow 0$, there must exist a subsequence $\left\{x_{n_{k}}\right\} \subset\left\{x_{n}\right\}$ such that $x_{n_{k}} \rightarrow x \in E(\lambda)$, then the problem $\lambda \in \Lambda$ is said to be generalized well-posed (in short G-wp);

(ii) If $E(\lambda)=\{x\}$ (a singleton), $\forall \lambda_{n} \in \Lambda, \lambda_{n} \rightarrow \lambda, \forall x_{n} \in E\left(\lambda_{n}, \epsilon_{n}\right), \epsilon_{n}>0$ with $\epsilon_{n} \rightarrow 0$, there must have $x_{n} \rightarrow x$, then $\lambda \in \Lambda$ is said to be well-posed (in short wp).

By Definitions 3.1, 3.2 and 3.3, it is easy to check the following.

\section{Lemma 3.2}

(1) If the problem $\lambda \in \Lambda$ is G-wp, then $\lambda$ must be GLP-wp.

(2) If the problem $\lambda \in \Lambda$ is wp, then $\lambda$ must be LP-wp.

\section{Lemma 3.3}

(1) If the problem $\lambda \in \Lambda$ is G-wp, then $\lambda$ must be GH-wp.

(2) If the problem $\lambda \in \Lambda$ is wp, then $\lambda$ must be $H$-wp.

\section{Sufficient conditions for well-posedness of (GVEP)}

Assume that the bounded rationality model $M=\{\Lambda, X, f, \Phi\}$ for (GVEP) is given. Now, let $(X, d)$ be a compact metric space, $(Y,\|\cdot\|)$ be a Banach space, and $C$ be a nonempty, closed, convex, and pointed cone in $Y$ with apex at the origin and int $C \neq \emptyset$.

In order to show sufficient conditions for well-posedness of (VEP), we first give the following lemmas.

Lemma 4.1 $(\Lambda, \rho)$ is a complete metric space. 
Proof Let $\left\{\lambda_{n}=\left(\varphi_{n}, G_{n}\right)\right\}$ be any Cauchy sequence in $\Lambda$, then for any $\epsilon>0$, there is a positive integer $N$ such that for any $n, m \geq N$,

$$
\rho\left(\lambda_{n}, \lambda_{m}\right)=\sup _{(x, y) \in X \times X}\left\|\varphi_{n}(x, y)-\varphi_{m}(x, y)\right\|+\sup _{x \in X} h\left(G_{n}(x), G_{m}(x)\right)<\epsilon .
$$

Then, for any fixed $(x, y) \in X \times Y,\left\{\varphi_{n}(x, y)\right\}$ is a Cauchy sequence in $Y$, and $\left\{G_{n}(x)\right\}$ is a Cauchy sequence in $K(X)$. By Lemma $2.5,(K(X), h)$ is a complete spaces and $(Y,\|\cdot\|)$ is also complete spaces. It follows that there exist $\varphi(x, y) \in Y$ and $G(x) \in K(X)$ such that $\lim _{m \rightarrow \infty} \varphi_{m}(x, y)=\varphi(x, y)$ and $\lim _{m \rightarrow \infty} G_{m}(x)=G(x)$. Thus, for all $n \geq N$, we have

$$
\sup _{(x, y) \in X \times X}\left\|\varphi_{n}(x, y)-\varphi(x, y)\right\|+\sup _{x \in X} h\left(G_{n}(x), G(x)\right) \leq \epsilon .
$$

Next, we will prove that $\lambda=(\varphi, G) \in \Lambda$, thus $(\Lambda, \rho)$ is a complete metric space.

(i) For any open convex neighborhood $V$ of zero element in $Y$, there is a positive integer $n_{0}$ such that for all $x, y \in X$,

$$
\varphi(x, y) \in \varphi_{n_{0}}(x, y)+\frac{V}{3}
$$

and

$$
\varphi_{n_{0}}(x, y) \in \varphi(x, y)+\frac{V}{3} .
$$

Since $\lambda_{n_{0}}=\left(\varphi_{n_{0}}, G_{n_{0}}\right) \in \Lambda, \varphi_{n_{0}}$ is $C$-upper semicontinuous on $X \times X$, thus there are an open neighborhood of $U_{1}$ at $x$ and an open neighborhood of $U_{2}$ at $y$ such that

$$
\varphi_{n_{0}}\left(x^{\prime}, y^{\prime}\right) \in \varphi_{n_{0}}(x, y)+\frac{V}{3}-C, \quad \forall x^{\prime} \in U_{1}, y^{\prime} \in U_{2} .
$$

By (1), (2), and (3), we have

$$
\varphi\left(x^{\prime}, y^{\prime}\right) \in \varphi_{n_{0}}\left(x^{\prime}, y^{\prime}\right)+\frac{V}{3} \subset \varphi_{n_{0}}(x, y)+\frac{2}{3} V-C \subset \varphi(x, y)+V-C .
$$

It shows $\varphi: X \times X \rightarrow Y$ is $C$-upper semicontinuous on $X \times X$.

(ii) It is easy to check that $\varphi(x, x)=\mathbf{0}, \forall x \in X, \sup _{(x, y) \in X \times X}\|\varphi(x, y)\|<+\infty, G: X \rightrightarrows X$ is continuous on $X$ and $\forall x \in X, G(x)$ is a nonempty compact set.

(iii) Since $\lambda_{n}=\left(\varphi_{n}, G_{n}\right) \in \Lambda$, there exists $x_{n} \in X$ such that $x_{n} \in G_{n}\left(x_{n}\right)$ and $\varphi_{n}\left(x_{n}, y\right) \notin$ -int $C, \forall y \in G_{n}\left(x_{n}\right)$. Firstly, we may suppose that $x_{n} \rightarrow x$, since $X$ is a compact metric space. For all $n \geq N$,

$$
\begin{aligned}
h\left(G_{n}\left(x_{n}\right), G(x)\right) & \leq h\left(G_{n}\left(x_{n}\right), G\left(x_{n}\right)\right)+h\left(G\left(x_{n}\right), G(x)\right) \\
& \leq \epsilon+h\left(G\left(x_{n}\right), G(x)\right) .
\end{aligned}
$$

Note that $G$ is continuous on $X$, we have

$$
h\left(G\left(x_{n}\right), G(x)\right) \rightarrow 0 .
$$


By (4) and (5), we get

$$
\begin{aligned}
d(x, G(x)) & \leq d\left(x, x_{n}\right)+d\left(x_{n}, G_{n}\left(x_{n}\right)\right)+h\left(G_{n}\left(x_{n}\right), G(x)\right) \\
& =d\left(x, x_{n}\right)+h\left(G_{n}\left(x_{n}\right), G\left(x_{n}\right)\right) \rightarrow 0 .
\end{aligned}
$$

Hence, $x \in G(x)$.

Finally, we only need to prove that $\varphi(x, y) \notin-$ int $C, \forall y \in G(x)$. By way of contradiction, assume that there exists $y_{0} \in G(x)$ such that $\varphi\left(x, y_{0}\right) \in-\operatorname{int} C$. It shows that there exists an open convex neighborhood $V$ of zero element in $Y$ such that $\varphi\left(x, y_{0}\right)+V \subset-\operatorname{int} C$.

Since $\sup _{(x, y) \in X \times X}\left\|\varphi_{n}(x, y)-\varphi(x, y)\right\| \rightarrow 0$, there is a positive integer $N_{1}$ such that $\forall n \geq N_{1}$,

$$
\varphi_{n}(x, y) \in \varphi(x, y)+\frac{V}{2}, \quad \forall x, y \in X
$$

By virtue of $h\left(G_{n}\left(x_{n}\right), G(x)\right) \rightarrow 0$, there exists $y_{n} \in G_{n}\left(x_{n}\right)$ such that $y_{n} \rightarrow y_{0}$. Note that $\varphi: X \times X \rightarrow Y$ is $C$-upper semicontinuous on $X \times X$, then there exists a positive integer $N_{2}$ such that $\forall n \geq N_{2}$,

$$
\varphi\left(x_{n}, y_{n}\right) \in \varphi\left(x, y_{0}\right)+\frac{V}{2}-C
$$

Let $N=\max \left\{N_{1}, N_{2}\right\}, \forall n \geq N$, by (6) and (7), we have

$$
\varphi_{n}\left(x_{n}, y_{n}\right) \in \varphi\left(x_{n}, y_{n}\right)+\frac{V}{2} \subset \varphi\left(x, y_{0}\right)+V-C \subset-\operatorname{int} C-C \subset-\operatorname{int} C .
$$

This is a contradiction to $\varphi_{n}\left(x_{n}, y\right) \notin-\operatorname{int} C, \forall y \in G_{n}\left(x_{n}\right)$.

Lemma 4.2 $f: \Lambda \rightrightarrows X$ is an usco mapping.

Proof Since $X$ is a compact metric space, by Lemma 2.4, it suffices to show that Graph $(f)$ is closed, where $\operatorname{Graph}(f)=\{(\lambda, x) \in \Lambda \times X: x \in f(\lambda)\}$. That is to say, $\forall \lambda_{n} \in \Lambda, \lambda_{n} \rightarrow \lambda$, $\forall x_{n} \in f\left(\lambda_{n}\right), x_{n} \rightarrow x$, we need to show that $x \in f(\lambda)$.

In fact, for each $n=1,2,3, \ldots$, since $x_{n} \in f\left(\lambda_{n}\right)$, then there exists $x_{n} \in X$ such that $x_{n} \in$ $G_{n}\left(x_{n}\right)$. Let $\sup _{x \in X} h\left(G_{n}(x), G(x)\right)=\epsilon_{n}$ with $\epsilon_{n} \rightarrow 0$, there must be $h\left(G_{n}\left(x_{n}\right), G\left(x_{n}\right)\right) \leq \epsilon_{n}$. Since $x_{n} \in G_{n}\left(x_{n}\right)$, there exists $x_{n}^{\prime} \in G\left(x_{n}\right)$ such that $d\left(x_{n}, x_{n}^{\prime}\right) \leq \epsilon_{n}$. By

$$
d\left(x_{n}^{\prime}, x\right) \leq d\left(x_{n}^{\prime}, x_{n}\right)+d\left(x_{n}, x\right) \rightarrow 0
$$

we get $x_{n}^{\prime} \rightarrow x$. Note that set-value mapping $G$ is continuous on $X$, then

$$
h\left(G\left(x_{n}\right), G(x)\right) \rightarrow 0 .
$$

By (8) and (9), we get

$$
d(x, G(x)) \leq d\left(x, x_{n}^{\prime}\right)+d\left(x_{n}^{\prime}, G\left(x_{n}\right)\right)+h\left(G\left(x_{n}\right), G(x)\right) \rightarrow 0 .
$$

Since $G(x)$ is a nonempty compact subset of $X$, by (10), we have $x \in G(x)$. It shows that $x \in f(\lambda)$. 
Lemma 4.3 For all $(\lambda, x) \in \Lambda \times X, \Phi(\lambda, x)$ is lower semicontinuous at $(\lambda, x)$.

Proof By Lemma 4.1, it is only need to show that $\forall \epsilon>0, \forall \lambda_{n}=\left(\varphi_{n}, G_{n}\right) \in \Lambda, \lambda_{n} \rightarrow \lambda=$ $(\varphi, G) \in \Lambda, \forall x_{n} \in X, x_{n} \rightarrow x \in X$, there exists a positive integer $N$ such that $\forall n \geq N$,

$$
\Phi\left(\lambda_{n}, x_{n}\right)>\Phi(\lambda, x)-\epsilon
$$

By definition of the least upper bound, there exists $y_{0} \in G(x)$ such that

$$
-\xi_{e}\left(\varphi\left(x, y_{0}\right)\right)>\Phi(\lambda, x)-\frac{\epsilon}{2}
$$

Note that $\sup _{x \in X} h\left(G_{n}(x), G(x)\right) \rightarrow 0$ and $h\left(G\left(x_{n}\right), G(x)\right) \rightarrow 0$, we have

$$
h\left(G_{n}\left(x_{n}\right), G(x)\right) \leq h\left(G_{n}\left(x_{n}\right), G\left(x_{n}\right)\right)+h\left(G\left(x_{n}\right), G(x)\right) \rightarrow 0 .
$$

From (13), there exists $y_{n} \in G_{n}\left(x_{n}\right)$ such that $d\left(y_{n}, y_{0}\right) \rightarrow 0$.

Since $\sup _{(x, y) \in X \times X}\left\|\varphi_{n}(x, y)-\varphi(x, y)\right\| \rightarrow 0$, that is to say, there exists a positive integer $N_{1}$ such that $\forall n \geq N_{1}$,

$$
\varphi_{n}\left(x_{n}, y_{n}\right)-\varphi\left(x_{n}, y_{n}\right) \in r e,
$$

where $r \in]-\frac{\epsilon}{4}, \frac{\epsilon}{4}[, e \in$ int $C$, $r e$ is an open neighborhood of zero element in $Y$. Thus, by (14) and Lemma 2.1(ii), we get

$$
-\frac{\epsilon}{4} \leq \xi_{e}\left(\varphi_{n}\left(x_{n}, y_{n}\right)-\varphi\left(x_{n}, y_{n}\right)\right) \leq \frac{\epsilon}{4} .
$$

By (15) and Lemma 2.1(iii), we get

$$
\begin{aligned}
-\xi_{e}\left(\varphi_{n}\left(x_{n}, y_{n}\right)\right) & =-\xi_{e}\left(\varphi_{n}\left(x_{n}, y_{n}\right)-\varphi\left(x_{n}, y_{n}\right)+\varphi\left(x_{n}, y_{n}\right)\right) \\
& \geq-\xi_{e}\left(\varphi\left(x_{n}, y_{n}\right)\right)-\xi_{e}\left(\varphi_{n}\left(x_{n}, y_{n}\right)-\varphi\left(x_{n}, y_{n}\right)\right) \\
& \geq-\xi_{e}\left(\varphi\left(x_{n}, y_{n}\right)\right)-\frac{\epsilon}{4}
\end{aligned}
$$

By Lemma 2.2, $\xi_{e} \circ \varphi$ is upper semicontinuous on $X \times X$. Then there exists a positive integer $N_{2}$ such that $\forall n \geq N_{2}$,

$$
-\xi_{e}\left(\varphi\left(x_{n}, y_{n}\right)\right)>-\xi_{e}\left(\varphi\left(x, y_{0}\right)\right)-\frac{\epsilon}{4}
$$

Let $N=\max \left\{N_{1}, N_{2}\right\}, \forall n \geq N$, by (16), (17), and (12), we get the inequality (11):

$$
\begin{aligned}
\Phi\left(\lambda_{n}, x_{n}\right) & =\sup _{y \in G_{n}\left(x_{n}\right)}\left\{-\xi_{e}\left(\varphi_{n}\left(x_{n}, y\right)\right)\right\} \geq-\xi_{e}\left(\varphi_{n}\left(x_{n}, y_{n}\right)\right) \\
& >-\xi_{e}\left(\varphi\left(x_{n}, y_{n}\right)\right)-\frac{\epsilon}{4}>-\xi_{e}\left(\varphi\left(x, y_{0}\right)\right)-\frac{\epsilon}{2}>\Phi(\lambda, x)-\epsilon .
\end{aligned}
$$

Next, we give sufficient conditions for G-wp and wp of (VEP). 


\section{Theorem 4.1}

(1) For all $\lambda \in \Lambda$, the problems $\lambda$ is G-wp.

(2) For all $\lambda \in \Lambda$, if $E(\lambda)=\{x\}$ (a singleton), then the problem $\lambda$ is $w p$.

Proof (1) $\forall \lambda_{n} \in \Lambda, \lambda_{n} \rightarrow \lambda, \forall x_{n} \in E\left(\lambda_{n}, \epsilon_{n}\right), \epsilon_{n}>0$ with $\epsilon_{n} \rightarrow 0$, then

$$
d\left(x_{n}, f\left(\lambda_{n}\right)\right) \leq \epsilon_{n}
$$

and

$$
\Phi\left(\lambda_{n}, x_{n}\right) \leq \epsilon_{n}
$$

From (18), there exists $u_{n} \in f\left(\lambda_{n}\right)$ such that $d\left(u_{n}, x_{n}\right) \rightarrow 0$ as $n \rightarrow \infty$. It follows by Lemma 4.2 and Lemma 2.3 that there exists $\left\{u_{n_{k}}\right\} \subset\left\{u_{n}\right\}$ such that $u_{n_{k}} \rightarrow x \in f(\lambda)$. By

$$
d\left(x_{n_{k}}, x\right) \leq d\left(x_{n_{k}}, u_{n_{k}}\right)+d\left(u_{n_{k}}, x\right) \rightarrow 0,
$$

we get

$$
x_{n_{k}} \rightarrow x \in f(\lambda) \text {. }
$$

By Lemma 4.3 and (19), we have

$$
0 \leq \Phi(\lambda, x) \leq \liminf _{n_{k} \rightarrow \infty} \Phi\left(\lambda_{n_{k}}, x_{n_{k}}\right) \leq \liminf _{n_{k} \rightarrow \infty} \epsilon_{n_{k}}=0 .
$$

That is,

$$
\Phi(\lambda, x)=0 .
$$

By (20) and (21), we have $x \in E(\lambda)$. It shows that $\lambda$ is G-wp.

(2) By way of contradiction. If the sequence $\left\{x_{n}\right\}$ does not converge $x$, then there exists an open neighborhood $O$ at $x$ and a subsequence $\left\{x_{n_{k}}\right\}$ of $\left\{x_{n}\right\}$ such that $x_{n_{k}} \notin O$. Since $E(\lambda)=\{x\}$ (a singleton), by the proof of (1), we get $x_{n_{k}} \rightarrow x$. This is a contradiction to $x_{n_{k}} \notin O$.

Similarly, by Lemmas 3.2 and 3.3, it is easy to check the following.

\section{Theorem 4.2}

(1) For all $\lambda \in \Lambda$, the problems $\lambda$ must be GLP-wp and GH-wp.

(2) For all $\lambda \in \Lambda$, if $E(\lambda)=\{x\}$ (a singleton), then the problem $\lambda$ must be LP-wp and $H$-wp.

Finally, we apply these results to (GEP). Let $Y=\mathbb{R}, C=[0,+\infty[$, the problem space of (GEP) is defined as

$$
\Lambda^{\prime}=\left\{\begin{array}{ll}
\lambda=(\varphi, G): \begin{array}{l}
\varphi \times X \times X \rightarrow \mathbb{R} \text { is upper semicontinuous on } X \times X, \\
\forall x \in X, \varphi(x, x)=0, \sup _{(x, y) \in X \times X}|\varphi(x, y)|<+\infty, \\
G: X \rightrightarrows X \text { is continuous with nonempty compact value on } X, \\
\exists x \in X \text { such that } x \in G(x) \text { and } \varphi(x, y) \geq 0, \forall y \in G(x) .
\end{array}
\end{array}\right\}
$$


For any $\lambda_{1}=\left(\varphi_{1}, G_{1}\right), \lambda_{2}=\left(\varphi_{2}, G_{2}\right) \in \Lambda^{\prime}$, define

$$
\varrho\left(\lambda_{1}, \lambda_{2}\right):=\sup _{(x, y) \in X \times X}\left|\varphi_{1}(x, y)-\varphi_{2}(x, y)\right|+\sup _{x \in X} h\left(G_{1}(x), G_{2}(x)\right) .
$$

It is easy to check that $\left(\Lambda^{\prime}, \varrho\right)$ is a complete metric space. Hence, we have the following.

\section{Corollary 4.1}

(1) For all $\lambda \in \Lambda^{\prime}$, the problem $\lambda$ is $G$-wp.

(2) For all $\lambda \in \Lambda^{\prime}$, if $E(\lambda)=\{x\}$ (a singleton), then the problem $\lambda$ is $w p$.

\section{Corollary 4.2}

(1) For all $\lambda \in \Lambda^{\prime}$, the problem $\lambda$ must be GLP-wp and GH-wp.

(2) For all $\lambda \in \Lambda^{\prime}$, if $E(\lambda)=\{x\}$ (a singleton), then the problem $\lambda$ must be LP-wp and H-wp.

\section{Competing interests}

The authors declare that they have no competing interests.

\section{Authors' contributions}

All authors contributed equally to the writing of this paper. All authors read and approved the final manuscript.

\section{Acknowledgements}

This research was supported by NSFC (Grant Number: 11161008), the Guizhou Provincial Science and Technology Foundation (20132235), (20122289). The authors thank the anonymous referees for their constructive comments which help us to revise the paper.

Received: 10 December 2013 Accepted: 17 March 2014 Published: 28 Mar 2014

\section{References}

1. Dontchev, AL, Zolezzi, T: Well-Posed Optimization Problems, vol. 1543. Springer, Berlin (1993)

2. Revalski, JP, Zhivkov, NV: Well-posed constrained optimization problems in metric spaces. J. Optim. Theory Appl. 76, 145-163 (1993)

3. Yang, H, Yu, J: Unified approaches to well-posedness with some applications. J. Glob. Optim. 31, 371-381 (2005)

4. Yu, J, Yang, $\mathrm{H}, \mathrm{Yu}, \mathrm{C}$ : Well-posed Ky Fan's point, quasi-variational inequality and Nash equilibrium problems. Nonlinear Anal. TMA 66, 777-790 (2007)

5. Zolezzi, T: Extended well-posedness of optimization problems. J. Optim. Theory Appl. 91, 257-266 (1996)

6. Huang, XX: Extended well-posedness properties of vector optimization problems. J. Optim. Theory Appl. 106, $165-182(2000)$

7. Huang, XX: Extended and strongly extended well-posedness of set-valued optimization problems. Math. Methods Oper. Res. 53, 101-116 (2001)

8. Huang, XX: Pointwise well-posedness of perturbed vector optimization problems in a vector-valued variational principle. J. Optim. Theory Appl. 108, 671-686 (2001)

9. Giannessi, F (ed.): Vector Variational Inequalities and Vector Equilibria: Mathematical Theories. Kluwer Academic, Dordrecht (2000)

10. Chen, GY, Huang, XX, Yang, XQ: Vector Optimization: Set-Valued and Variational Analysis. Springer, Berlin (2005)

11. Miglierina, E, Molho, E, Rocca, M: Well-posedness and scalarization in vector optimization. J. Optim. Theory Appl. 126 391-409 (2005)

12. Durea, M: Scalarization for pointwise well-posed vectorial problems. Math. Methods Oper. Res. 66, 409-418 (2007)

13. Crespi, GP, Guerraggio, A, Rocca, M: Well posedness in vector optimization problems and vector variational inequalities. J. Optim. Theory Appl. 132, 213-226 (2007)

14. Huang, XX, Yang, XQ: Levitin-Polyak well-posedness of constrained vector optimization problems. J. Glob. Optim. 37, 287-304 (2007)

15. Zui, X, Zhu, DL, Huang, XX: Levitin-Polyak well-posedness in generalized vector variational inequality problem with functional constraints. Math. Methods Oper. Res. 67, 505-524 (2008)

16. Li, Z, Xia, FQ: Scalarization method for Levitin-Polyak well-posedness of vectorial optimization problems. Math. Methods Oper. Res. 76, 361-375 (2012)

17. Li, SJ, Li, MH: Levitin-Polyak well-posedness of vector equilibrium problems. Math. Methods Oper. Res. 69, 125-140 (2009)

18. Gerth, C, Weidner, P: Nonconvex separation theorems and some applications in vector optimization. J. Optim. Theory Appl. 67, 297-320 (1990)

19. Anderlini, L, Canning, D: Structural stability implies robustness to bounded rationality. J. Econ. Theory 101, 395-422 (2001) 
20. Yu, C, Yu, J: On structural stability and robustness to bounded rationality. Nonlinear Anal. TMA 65, 583-592 (2006)

21. $Y u, C, Y u, J$ : Bounded rationality in multiobjective games. Nonlinear Anal. TMA 67, 930-937 (2007)

22. Yu, J, Yang, H, Yu, C: Structural stability and robustness to bounded rationality for non-compact cases. J. Glob. Optim. 44, 149-157 (2009)

23. Luc, DT: Theory of Vector Optimization. Springer, Berlin (1989)

24. Aliprantis, CD, Border, KC: Infinite Dimensional Analysis. Springer, Berlin (1999)

25. Klein, E, Thompson, AC: Theory of Correspondences. Wiley, New York (1984)

10.1186/1029-242X-2014-127

Cite this article as: Deng and Xiang: Well-posed generalized vector equilibrium problems. Journal of Inequalities and Applications 2014, 2014:127

Submit your manuscript to a SpringerOpen ${ }^{\odot}$ journal and benefit from:

- Convenient online submission

- Rigorous peer review

- Immediate publication on acceptance

- Open access: articles freely available online

- High visibility within the field

- Retaining the copyright to your article 